\section{Evidence-Based Neuroimaging Diagnosis and Treatment: Improving the Quality of Neuroimaging in Patient Care}

\author{
L.S. Medina, P.C. Sanelli, and J.G. Jarvik, Eds.
}

New York, NY: Springer, 2013, 650 pages, $\$ 189$

This interesting book is one of a series that aims to provide a systematic framework for understanding imaging choices based on evidence reported in the literature. Written mostly by radiologists and clinicians, the book is intended to be a reference for decision making in clinical practice and contains information on the use of neuroimaging in every possible clinical case of brain, spine, and head and neck disorders. The imaging modalities that are discussed include CT, MR imaging, MR angiography, SPECT (mostly of the spine), and PET (mostly of head and neck masses). On the basis of the clinical literature as evaluated from cost-effectiveness-analysis and evidence-based-medicine perspectives, each chapter poses questions such as "in this condition, which imaging method is warranted, and why?" and then answers those questions. The authors pay most attention to the results of clinical trials that did or did not include neuroimaging options, attempting to prove or disprove the value of neuroimaging studies in the outcomes of patients with cerebrovascular diseases, spine disorders, or head and neck cancers. The authors also predict the future value of newer neuroimaging methods that may soon be used to evaluate some of the more incomprehensible and intractable pediatric neurodevelopmental and neurodegenerative diseases. Interestingly, every chapter has a section that describes further research needed to optimize the use of current neuroimaging methods or, in the case of MR imaging, to expand its use to newer sequences. Almost all the chapters include physiologic imaging as an example of such a modality, along with the use of SPECT and PET for seizure disorders; bone SPECT for spinal injection for low back pain; PET for brain cancer, neck masses, neck adenopathy, and diagnosis of cervical lymph node metastasis in head and neck cancer; and MR spectroscopy.

Readers can easily refer to this book for the answers to probable clinical questions and supporting evidence for those answers. Readers can also use the "Key Points" sections to quickly determine whether a certain modality, such as PET or SPECT, is dealt with in those questions. The chapters are in a hierarchical format that allows readers to quickly find specific questions and answers of interest and then broaden their search if desired. The consistency in the depth and style of each chapter will assure readers that each is consistently informative and credible. For nuclear medicine physicians, the information on CT, MR imaging, and angiography for brain, spine, and head and neck disorders will help them grasp the big picture on the current best practice and changes expected in the near future. The only area that is lacking is information about nuclear medicine imaging - a surprising shortcoming considering the comprehensiveness of the book, and I frowned to see that

COPYRIGHT (c) 2014 by the Society of Nuclear Medicine and Molecular Imaging, Inc. many chapters described ${ }^{18}$ F-FDG PET using just the generic term $P E T$. This lack may have occurred because the literature does not include enough publications with sound evidence supporting the use of nuclear medicine imaging in clinical decision making, which would be required in a book on evidence-based medicine such as this one. Another possibility is that the authors simply chose not to describe evidence in the field of nuclear medicine. For example, in the chapter on seizure disorders, ${ }^{18} \mathrm{~F}$-FDG PET is cited with less frequency than in the literature, and in the chapters on acute ischemic stroke and atherosclerotic disease of the cervical carotid artery, I would have expected acetazolamide stress SPECT (either ${ }^{123} \mathrm{I}-\beta$-methyliodophenylpentadecanoic acid or ${ }^{99 \mathrm{~m}} \mathrm{Tc}-$ hexamethylpropyleneamine oxime) to be described but it was entirely left out. Likewise, the chapter on brain cancer neglected to mention ${ }^{11} \mathrm{C}$-methionine PET. Omitting evidence-based information on various uses of SPECT and PET warrants the issuing of a new edition that is truly about neuroimaging instead of merely radiologic neuroimaging.

This book would be best utilized by clinicians who are interested in fields other than their own, such as a neurovascular expert who is interested in epilepsy or neurodevelopmental disorders (attention deficit-hyperactivity disorder or autism spectrum disorder) or a nuclear medicine physician who is interested in neuroimaging of patients undergoing endovascular treatment for acute ischemic stroke or patients undergoing spinal injections for low back pain. If readers are interested in an in-depth, balanced interpretation of their own field, they may find other, more specialized references and books preferable to this one. However, this book is the right one for readers interested in how to collect and combine a database to understand the currently optimal, recognized clinical use of a neuroimaging modality and for readers interested in the scientific background for the state-of-the-art use of CT, MR imaging, and angiography. The beauty of this book is that parts I and II describe the principles of evidence-based imaging and neuroimaging in the radiologic field and compose an ideal introduction to the remainder of the book. Part II imparts knowledge about the decision-support process, as well as covering how best to use neuroimaging as a screening tool and how to handle any incidental findings. Also detailed is how to comply with the economic and regulatory changes in the United States-information that readers in Asia and Europe can apply by analogy to their own regulatory situation.

I believe that the timing of this book is perfect from the perspective of health care reform in the United States, because neuroimaging is as yet neither an integrative diagnostic procedure (unlike auscultation or palpation) nor an independent procedure. Neuroimaging is costly in both money and time, and its use in clinical practice requires sufficient supporting evidence to persuade layperson policy makers and referring physicians. If we, the nuclear medicine physicians, want our nuclear neuroimaging to be used more often by clinicians and approved by the health-care system, we now know what data we should collect and publish so that secondary literature such as the Cochrane Library or this type of book will reflect our consensus. Reading this book cover to cover was an enlightening adventure for me, educational yet sometimes confusing or frustrating. Certain terms are abbreviated differently 
in each chapter, mention of certain nuclear neuroimaging modalities is neglected a few times, and certain important references or review articles on SPECT/CT and PET/CT are omitted.

Summing up, there can be a prospective study in which PET/CT is performed immediately after clinical evaluation and then the incremental value of adding MR imaging is determined. Taking the value of CT for granted and trying to prove the incremental value of PET/CT might not always be justified when PET/CT is a single technique and PET/MR imaging is a hybrid technique awaiting validation of its clinical efficacy over time. What we call comparative effectiveness research might replace the present concept in which imaging studies are clinically validated one at a time; that is to say, first CT, then MR imaging, and then PET. After the full employment of hybrid imaging technologies, the present custom of our medical community to try to prove incremental value will become obsolete.

\section{Dong-Soo Lee}

Seoul National University

28 Yungundong Chonnogu

Seoul 110-744, Korea

E-mail:dsl@snu.ac.kr

Published online Apr. 3, 2014

DOI: 10.2967/jnumed.114.139287

\section{Erratum}

There were errors in the reprint permission statements for Figures 2C and 2D in "Acoustic and Photoacoustic Molecular Imaging of Cancer," by Wilson et al. (J Nucl Med. 2013;54:1851-1854). Figure 2C was reproduced with the permission of the American Chemical Society from Homan KA, Souza M, Truby R, et al. Silver nanoplate contrast agents for in vivo molecular photoacoustic imaging. ACS Nano. 2012;6:641-650. Figure 2D was reproduced with the permission of the Radiological Society of North America from Herzog E, Taruttis A, Beziere N, Lutich AA, Razansky D, Ntziachristos V. Optical imaging of cancer heterogeneity with multispectral optoacoustic tomography. Radiology. 2012;263:461-468. We regret the errors. 